\title{
REGISTRATION OF 2D DRAWINGS ON A 3D POINT CLOUD AS A SUPPORT FOR THE MODELING OF COMPLEX ARCHITECTURES
}

\author{
Q. Semler ${ }^{1}$, D. Suwardhi ${ }^{2}$, E. Alby ${ }^{1}$, A. Murtiyoso ${ }^{1}$, H. Macher ${ }^{1}$. \\ ${ }^{1}$ Photogrammetry and Geomatics Group, ICube Laboratory UMR 7357 INSA Strasbourg, France \\ - (quentin.semler,emmanuel.alby,arnadi.murtiyoso,helene.macher)@insa-strasbourg.fr \\ ${ }^{2}$ Remote Sensing and GIS Group, Bandung Institute of Technology, Indonesia - deni@gd.itb.ac.id
}

\section{Commission II, WG II/8}

KEY WORDS: Architectural drawings, semantics, discretization, segmentation by slices, ICP, registration, buffer zone, classification.

\begin{abstract}
:
Laser scanning and photogrammetry methods have seen immense development in the last years. From bulky inaccessible systems, these two 3D recording systems has become more or less ubiquitous, which is also the case in the heritage domain. However, automation in point cloud classification and semantic annotation remains a much studied topic. In this paper, an approach to help the classification of point cloud is presented using the help of existing 2D drawings. The 2D drawings are registered unto the 3D data, to then be used as a support in the 3D modeling step. The developed approach includes the computation of the point cloud cross section and detection of feature points. This is then used in a 3D transformation followed by ICP refinement to properly register the vectorized $2 \mathrm{D}$ drawing on the $3 \mathrm{D}$ point cloud. Results show that the developed algorithm manages to register the $2 \mathrm{D}$ drawing automatically and with promising results. The automatically registered $2 \mathrm{D}$ drawing, which often times already includes semantic information, was then used to help classify the point cloud into several architectural classes.
\end{abstract}

\section{INTRODUCTION}

Long time considered as inaccessible for small companies, nowadays laser scanning and photogrammetry are increasingly democratized (Murtiyoso et al., 2017). Data can be quickly collected in the field with a high level of accuracy (Remondino, 2011). In addition, there is now a few software and algorithms (Berger et al., 2014) easily accessible to automate the processing. However, point clouds processing still remain particularly time-consuming (Macher et al., 2017, Tang et al., 2010). This is particularly the case for old buildings with rich architectural details (Antonopoulos, Antonopoulou, 2017, Murphy et al., 2013, Murtiyoso, Grussenmeyer, 2019). To accelerate the 3D modeling process based on point cloud, our experiment will propose to introduce $2 \mathrm{D}$ CAD files. Today many building plans are already in digital format and old drawing paper can easily be vectorized. These 2D layout drawings can serve as a base for modeling by using the semantic aspect that is already available in the drawing (usually building elements are stored in separate layers according to their semantic information). Furthermore, the point cloud can serve as a geometric reference. This leads to a research question about the characteristics of a 2D drawing, its precision and its interest for 3D modeling. Several issues related to data formatting, dimension management and semantic extraction will be discussed in this paper.

Some previous work can be identified in the literature, although most relies on georeferenced GIS (Geographical Information System) files. This includes a study by (Liu et al., 2018), which uses GIS data to help the segmentation of a 3D point cloud. (Murtiyoso, Grussenmeyer, 2019) adds semantic annotation to this approach by acquiring the attributes as stored in the GIS file. Another approach developed by (Kaiser et al., 2017) used a similar method employed to aerial images.

\section{DATA USED FOR THE STUDY}

\subsection{Building history}
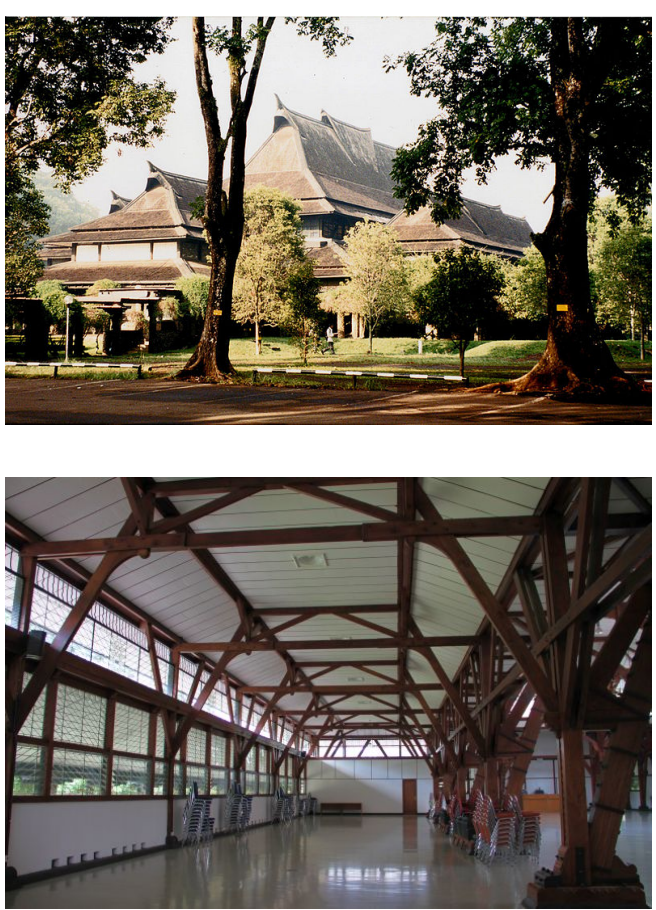

Figure 1. Photographs of "Aula Barat" building, Bandung Institute of Technology (2015)

The building used for the case study is located in the Bandung Institute of Technology in Indonesia. The construction is called Aula Barat, meaning Western Hall, which is famous 
for its colonial Art Deco style architecture. The building was designed by the Dutch architect Henri MacLaine Pont in the beginning of the 20th century. The remarkable aspects of the monument are its large area $\left(2300 \mathrm{~m}^{2}\right)$ and the complexity of its mix of traditional Indonesian and modern European joisted roofs (Figure 1). The western lobby has not changed since its initial construction except for some minor repairs. The hypothesis of a possible change can therefore be set aside for the rest of the study.

\subsection{Initial data}

The data employed for this project come from different sources which have been produced and acquired at several periods. For this project, we used a 3D point cloud which is the result of a laser scanning acquisition. As described above, we also use architectural drawings vectorized in AutoCAD format from 2D paper drawings. For this study, we only use the floor plan. The integration of the elevation or roof drawings could be studied in a complementary way.

\section{PREPROCESSING}

In order to avoid problems related to the use of many software, we decided to develop all algorithms in MATLAB. The first step focuses on conditioning the input data and a look on the constraints imposed by the problem. The preprocessing involves the cleaning and correction of the architectural plan, as described in (Horna et al., 2007). The idea is to be able to control the topology of the objects and create a good management of the layers as created by the architect. In addition, the layers of interest can be identified and pre-selected at this stage of the processing. For instance, dimensions or text layers are not necessary for further processing. This step allows to introduce valid, consistent and non-repetitive data for the rest of the process.

The second step is the discretization of linear objects in order to register the 3D point cloud and architectural plan with the employed registration algorithms such as ICP (Iterative Closest Point) described in (Besl, McKay, 1992). This approach will therefore be different from the one proposed by (Alshawa, 2007) which is based on the search for geometric primitives in the point cloud (Daniels et al., 2007). The main issue with the division of CAD entities (line, polyline, circle, etc.) into point entity is the value of the step that the user must define to approach the object. The latter must be consistent with the 3D cloud sampling. The programming of a function in VBA for
AutoCad made it possible and must be in compliance with the following criteria :

- Maintain the vertices of the object,

- Ask the user for the desired division step. Thanks to this, it is possible to match the sample of the $2 \mathrm{D}$ drawing with the $3 \mathrm{D}$ point cloud,

- Keep the semantic information of the object contained in the layer,

- Be sufficiently complete to work with several different kinds of entities regularly used by an architect (line, polyline, and circle).

\section{REGISTRATION OF THE ARCHITECTURAL DRAWING}

The registration of a plan (2D space) with a $3 \mathrm{D}$ point cloud is not straight forward. Drawings remain, more or less, a faithful representation of a three dimensional object. Therefore, they can at first glance correspond to several altitudes of the cloud at the same time. Thus, the floor plan can be registered to varying heights ranging from the floor to the ceiling. This ambiguity, without more preprocessing, prevents a proper registration and thus provides inconsistent results (Figure 2 / LEFT).

To address this issue, we segmented the $3 \mathrm{D}$ point cloud into 198 regular horizontal slices of $10 \mathrm{~cm}$ thickness. The aim is to approach the two-dimensional space of the floor plan with the horizontal slices of the point cloud. The comparison of the successive positions of the $2 \mathrm{D}$ plan on the different sections allow to identify the areas of interest useful for the registration. To obtain quantifiable criterion of the quality of our positioning in the most automatic way possible, the ICP algorithm was used. It is employed to minimize the distance between two clouds of points iteratively. This approach provides a quality index related to the registration for each cross-section (in the form of RMSE values). The section for which the discretized 2D drawing seems to match the best can then be determined from these values. A first solution was developed using all the semantics of the architectural plan without taking into account the nature of each entity. This method will then be refined with the entities providing the best results.

\subsection{First global registration}

This first approach is termed global in the sense that all the points of the $2 \mathrm{D}$ drawing are used for registration, regardless

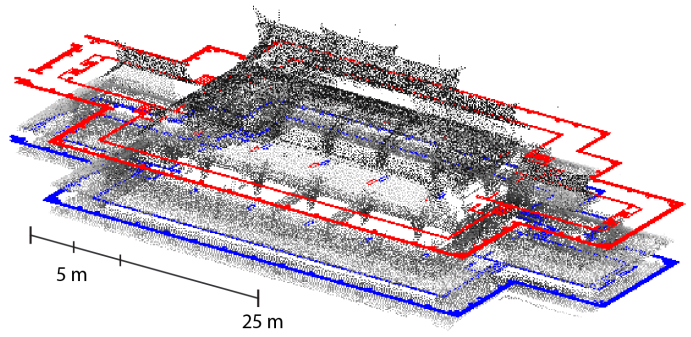

Figure 2. LEFT : First result of the registration of the discretized 2D plan on the 3D point cloud using ICP — RIGHT : Ambiguities related to the possible positions of the $2 \mathrm{D}$ drawing in the point cloud. 
of its semantics. The program goes through all the sections and records the root mean square error for each registration, respecting the workflow (Figure3).

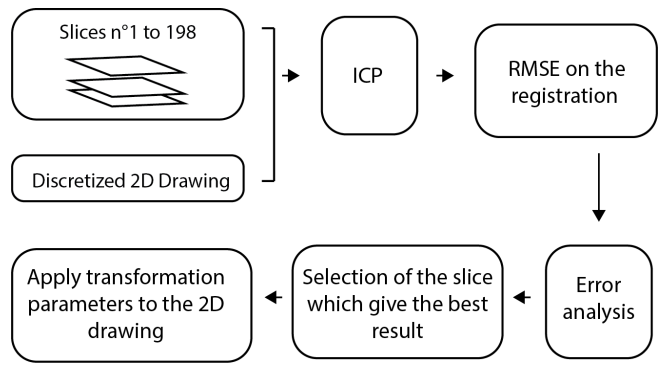

Figure 3. Processing chain for the registration of the architectural drawing with the point cloud.

To simplify the analysis of results, a graph is drawn up to represent the evolution of registration errors according to each slice. Thus, we can see which cuts match the best with the architectural drawing. Furthermore, we can indirectly observe the changes in geometry of the building and deduce the intervals of interest for the registration. The results obtained with this method are visible in (Figure 4).

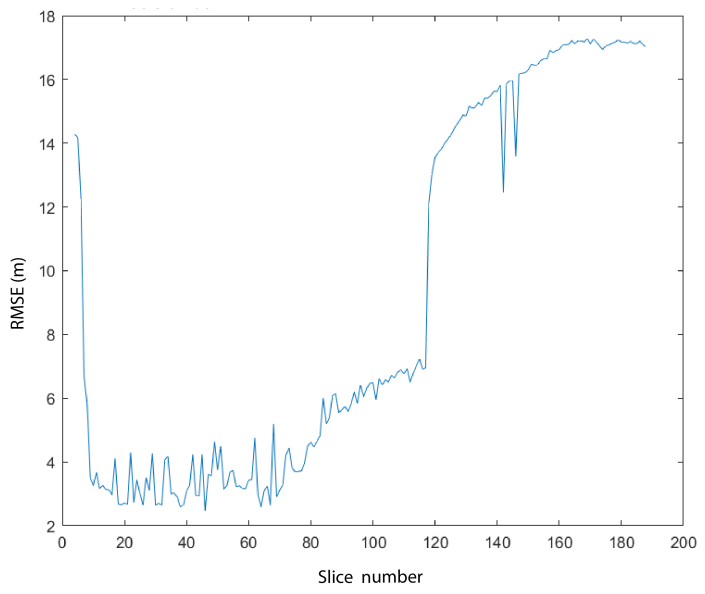

Figure 4. RMSE error on the registration depending on each slice in the global case

As expected, lower cross-sections provide better results, which seems to be quite normal since we use a ground plan as the input data. Significant residuals on slices from 1 to 10 are the result of noise on the point cloud. After slice 80, the residues increase sharply, which is explained by the fact that we go through sections of the roof. The transformation parameters used for the final registration of the $2 \mathrm{D}$ drawing are given by the slice with lowest residual after convergence. The best one is provided here by the slice 37 with a RMSE equal to $90 \mathrm{~cm}$. However, a visual analysis of the selected slice is required to verify that the algorithm has not converged to a local minimum or it has not been biased by other factors. The results obtained are consistent with the point cloud and overcame the dimension issue as illustrated in the beginning of this section with (Figure $2 / L E F T$ ). To conclude, the results are acceptable but the lowest error (RMSE) remains important for our study. If the registration is too approximative, the quality of the cloud classification will be affected later. Therefore, we try to refine the results obtained with new considerations. Indeed, we subsequently used all the semantics in the architectural plan. However, some semantics are more reliable than others which is why we added a filter which may help reach the best registration results.

\subsection{Refined registration}

Afterwards, we try to improve the previous result using a semantic filter. The selection of entities, which are useful for the registration will lead to better results. For instance, the walls layer is in most cases more reliable for the registration than stairs . A thorough study has therefore allowed to select the entities useful to find the optimum registration and altitudes of interest for each semantics. The same workflow (Figure 3) have been applied with the sole exception being the input data. Discretized 2D drawing has been decomposed by semantics in order to analyse the impact of this separation on ICP algorithm. The results are presented in (Figure 5).
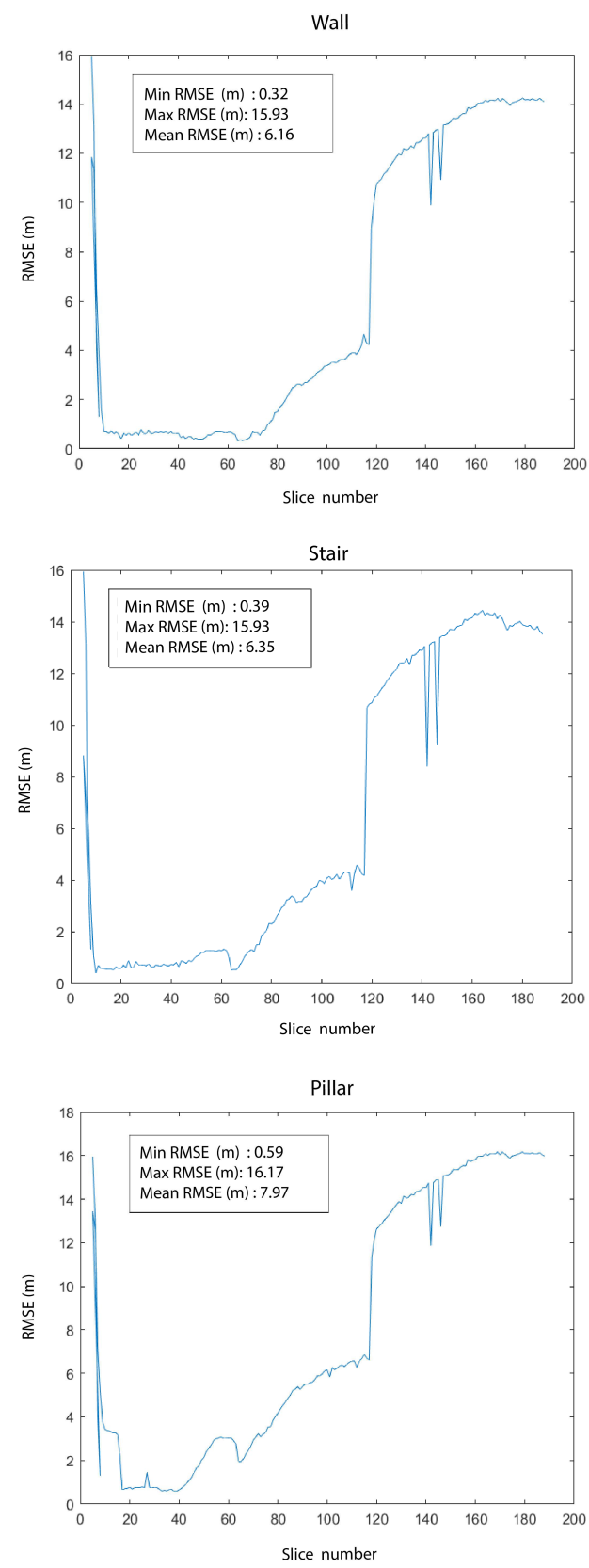

Figure 5. RMSE error on the registration depending on semantic of the data 
Using points which represent the walls in the architectural plan gave better results than in the previous experiment. The lowest RMSE is now $30 \mathrm{~cm}$ (the previous value was $90 \mathrm{~cm}$ ). Again, the residuals are fine for the entire lower part of the building and have the advantage of being much more stable between sections number 10 to 75 . The stairs layer also give a rather satisfactory result. The curve is generally close to that obtained with the use of the walls layer. However RMSE remain higher on average. In addition, it is necessary to be vigilant about the analysis of this result produced because the registration remains correct on higher slices where stairs are not represented anymore in the point cloud. The curve obtained with the pillars is, on the contrary, rather disappointing. Indeed, it could be expected that the pillars, like the walls, would be a precise structural element for registration. However, the latter offers acceptable residuals over a shorter interval with a $60 \mathrm{~cm}$ minimum RMSE. It could be explained by the elaborate geometry of pillars. More complex than a wall, they are composed of more than a single vertical component.

From this simple semantic threshold, we can see that the results are different from the result obtained without. The semantics "walls" offers a registration parameter with the lowest error and will be the best information to use for the registration here. The lowest residual is on slice 64. This cross-section, located at a height of about 6 meters, is rather high, contrary to what one might expect. However, the upper part of a room is often the best place to match a $2 \mathrm{D}$ drawing because it is a clear space with no furnitures or windows. A visual inspection can also confirm the registration. As the results are satisfactory, we can validate the final registration with its 3 translations $(X, Y, Z)$ and 3 rotations $(\omega, \Phi, \kappa)$. Then, these parameters must be applied to the entire $2 \mathrm{D}$ drawing.

\section{CLASSIFICATION OF THE POINT CLOUD}

As mentioned previously, the 2D layout can serve as a base for modeling by using the semantic aspect while the point cloud can serve as a geometric reference. After the registration, the proximity of the data is used to apply the semantic on the 3D point cloud. Buffer zones were created around each instance (Murtiyoso, Grussenmeyer, 2019). This will attribute to each cluster of the 3D cloud, its classification according to the $2 \mathrm{D}$ plan.

\subsection{Semantic extraction with buffer area}

In order to extract semantic information from the architectural plan, a circular buffer zone is defined around each point. The diameter of this area is defined by the user according to the specificity of the point cloud to be classified (spatial resolution, noise, etc.). This diameter may also vary depending on the nature of the entity. Thus, each point of a slice of the point cloud in this area will be assigned the semantics of the buffer zone. This classification is carried out iteratively on each slice.

By creating buffer zones of different sizes and sometimes intersecting, some points should have dual classification. In this case, they have to be rectified and reprocessed. The main issue is to know which of the semantics is most representative of this point. The choice was to compute the distance from each neighboring semantics. The closest semantic was finally applied on the point. To achieve more accurate and less noisy classification, the interval of slices to be processed can be set by the user. To define the stops to be used (according to the different semantics), we can imagine two solutions :

- Use the architectural elevation plans and read the necessary heights (not used in this study).

- Read the semantic registration graphs obtained in the previous section (4.2). Indeed, the appearance of the curve is modified when the geometry of the slice of the building changes. Interval of interest can then be determined.

Finally, we use the second solution. For instance, the following information can be deduced from (Figure $6 / L E F T$ ). As we employ a ground plan to perform the classification, we will introduce errors by keeping slices that concern the roofs. In the same way, sections representing noise under the cloud are not useful. Thus, from this graph, we will be able to select the most suitable interval on which to apply the buffer zone extraction algorithm. In this case, we use slices number 10 to 75 for "walls". The final parameters used for classification are defined in the (Table 1). The buffer distances were taken relatively wide to accommodate the spatial inaccuracies of the $2 \mathrm{D}$ plan. Indeed, it should not be forgotten in this study that the architectural plan, although close to the cloud, was drawn up before construction and therefore does not necessarily reflect
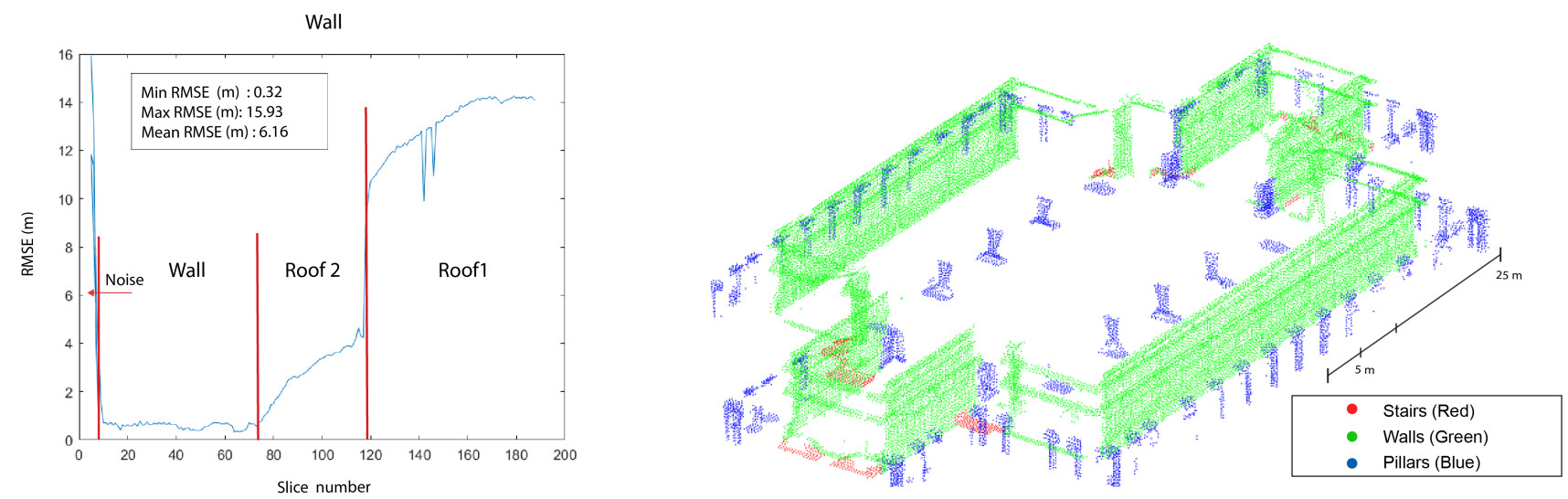

Figure 6. LEFT : Interval of interest for "walls" semantics — RIGHT : Classification results in 3 different classes (Walls,Stairs,Pillars). 
the reality of what has been built. With this method, $25 \%$ of the $3 \mathrm{D}$ point cloud was classified. This result is rather low but it seems visually quite consistent in (Figure $6 /$ RIGHT)). Most of the remaining points are either part of the roof or the ground and can be easily segmented as a second step.

\begin{tabular}{ccccc}
\hline Semantic & Low slice & High slice & $\begin{array}{c}\text { Buffer } \\
\text { distance }\end{array}$ & $\begin{array}{c}\text { Nb of classi- } \\
\text { fied points }\end{array}$ \\
\hline Walls & 10 & 75 & 0.5 & 30785 \\
Stairs & 10 & 15 & 0.5 & 1011 \\
Pillars & 10 & 40 & 0.6 & 8126 \\
\hline
\end{tabular}

Table 1. Parameters used for classification

\subsection{Evaluation of the classification}

A quick visual analysis of the (Figure 6 / LEFT) shows that the points are, correctly identified and having the correct semantics. In order to assess the quality of the classification, a manual segmentation was performed on a small portion of the classified cloud. This will serve as a reference data. Comparing the reference point cloud with the automatically extracted point cloud will allow us to determine the percentage of true positives and the percentage of false negatives for each semantics (Table 2).

\begin{tabular}{cccc}
\hline & Walls & Stairs & Pillars \\
\hline True Positive & $95 \%$ & $90 \%$ & $85 \%$ \\
False Negative & $4 \%$ & $3 \%$ & $7 \%$ \\
\hline
\end{tabular}

Table 2. Evaluation of the classification

The results are satisfactory with a good percentage of true positives. False positives are largely composed of ground points that do not currently included in our classification. A finer setting during processing or an additional step will probably solve the problem. Overall, the results obtained are therefore rather promising for the part of the cloud that has been classified.

\section{CONCLUSION}

To conclude, the process of semantic extraction developed in this study is promising. Compared to a manual classification, the amount of time saved associated with the use of this processing chain decreased, although it still depends on the complexity of the building and the semantics used. This processing illustrates that architectural and construction drawings can be useful for the purpose of 3D modeling. Some improvements can be made to this algorithm. It will be particularly interesting to add more information such as roof and elevations drawings of the building to reduce the number of unclassified points. Another possibility is the development of a hybrid treatment using the segmentation and classification of plan segments, as developed in (Boulassal et al., 2007, Macher et al., 2017). The application of this processing chain to other data sets could also be interesting. It can help to improve the robustness of the process.

\section{REFERENCES}

Alshawa, M., 2007. 1CL: Iterative closest line A novel point cloud registration algorithm based on linear features. Ekscentar, 53-59.

Antonopoulos, A., Antonopoulou, S., 2017. 3D survey and BIM-ready modelling of a Greek Orthodox Church in Athens. 23-25.

Berger, M., Tagliasacchi, A., Seversky, L., Alliez, P., Levine, J., Sharf, A., Silva, C., 2014. State of the art in surface reconstruction from point clouds. EUROGRAPHICS star reports, 1, $161-185$.

Besl, J., McKay, N., 1992. A method for Registration of 3-D shapes. IEEE Transaction on Pattern Analysis and MAnchine Intelligence, 239-255.

Boulassal, H., Landes, T., Grussenmeyer, P., Tarsha-Kurdi, F., 2007. Automatic segmentation of building facades using Terrestrial Laser Data. 65-70.

Daniels, J. I., Ha, L. K., Ochotta, T., Silva, C. T., 2007. Robust smooth feature extraction from point clouds. IEEE International Conference on Shape Modeling and Applications 2007 (SMI'07), IEEE, 123-136.

Horna, S., Damiand, G., Meneveaux, D., Bertrand, Y., 2007. Building 3D indoor scenes topology from 2D architectural plans. Proc. of 2nd International Conference on Computer Graphics Theory and Applications (GRAPP), 37-44.

Kaiser, P., Wegner, J. D., Lucchi, A., Jaggi, M., Hofmann, T., Schindler, K., 2017. Learning aerial image segmentation from online maps. IEEE Transactions on Geoscience and Remote Sensing, 55(11), 6054-6068.

Liu, C.-J., Krylov, V., Dahyot, R., 2018. 3d point cloud segmentation using gis. 20th Irish Machine Vision and Image Processing Conference, 41-48.

Macher, H., Landes, T., Grussenmeyer, P., 2017. From Point Clouds to Building Information Models: 3D Semi-Automatic Reconstruction of Indoors of Existing Buildings. Applied Sciences, 7(1030), 1-30.

Murphy, M., McGovern, E., Pavia, S., 2013. Historic Building Information Modelling-Adding intelligence to laser and image based surveys of European classical architecture. ISPRS journal of photogrammetry and remote sensing, 76, 89-102.

Murtiyoso, A., Grussenmeyer, P., 2019. Point cloud segmentation and semantic annotation aided by GIS data for heritage complexes. The International Archives of the Photogrammetry, Remote Sensing and Spatial Information Sciences, 42, 163170.

Murtiyoso, A., Koehl, M., Grussenmeyer, P., Freville, T., 2017. Acquisition And Processing Protocols For Uav Images: 3d Modeling Of Historical Buildings Using Photogrammetry. ISPRS Annals of Photogrammetry, Remote Sensing \& Spatial Information Sciences, 4, 163-170.

Remondino, F., 2011. Heritage recording and 3D modeling with photogrammetry and 3D scanning. Remote Sensing, 3(6), 1104-1138.

Tang, P., Huber, D., Akinci, B., Lipman, R., Lytle, A., 2010. Automatic reconstruction of as-built building information models from laser-scanned point clouds: A review of related techniques. Automation in construction, 19(7), 829-843. 